\title{
LETTER \\ Energy-Efficient Connectivity Re-Establishment in UASNs with Dumb Nodes
}

\author{
Qiuli CHEN ${ }^{\dagger, \dagger \dagger}$, Ming $\mathrm{HE}^{\dagger, \dagger \dagger a)}$, Fei DAI ${ }^{\dagger}$, Nonmembers, and Chaozheng $\mathrm{ZHU}^{\dagger}$, Student Member
}

SUMMARY The changes of temperature, salinity and ocean current in underwater environment, have adverse effects on the communication range of sensors, and make them become temporary failure. These temporarily misbehaving sensors are called dumb nodes. In this paper, an energyefficient connectivity re-establishment (EECR) scheme is proposed. It can reconstruct the topology of underwater acoustic sensor networks (UASNs) with the existing of dumb nodes. Due to the dynamic of underwater environment, the generation and recovery of dumb nodes also change dynamically, resulting in intermittent interruption of network topology. Therefore, a multi-band transmission mode for dumb nodes is designed firstly. It ensures that the current stored data of dumb nodes can be sent out in time. Subsequently, a connectivity re-establishment scheme of sub-nodes is designed. The topology reconstruction is adaptively implemented by changing the current transmission path. This scheme does't need to arrange the sleep nodes in advance. So it can reduce the message expenses and energy consumption greatly. Simulation results show that the proposed method has better network performance under the same conditions than the classical algorithms named LETC and A1. What's more, our method has a higher network throughput rate when the nodes' dumb behavior has a shorter duration.

key words: connectivity re-establishment, dumb nodes, data reliable transmission, underwater acoustic sensor networks (UASNs)

\section{Introduction}

Underwater acoustic sensor network (UASN) consists of underwater sensor nodes in a pre-deployed or self-organizing manner. The underwater nodes which have limited energy and low computational power, transmits the sensed information to surface sink via multi-hop manner. However, in the complex and changeable underwater environment, the topology has dynamic evolution. Environmental interference, nodes failure, and malicious attacks have posed the challenges for reliable data transmission. In this paper, we present a scheme for re-establishment of the topology which has been temporarily broken due to dumb nodes in underwater sensing. A sensor node is termed as dumb, when its communication range decreased due to adverse environmental effects such as current movement, high salinity and high temperature. Because of which, the dumb node can only sense the environment, but can't transmit information to any neighbors. This kind of behavior can be called as dumb

Manuscript received January 23, 2018.

Manuscript revised July 24, 2018.

Manuscript publicized August 20, 2018.

The authors are with the Army Engineering University of PLA, Nanjing 210007, China.

${ }^{\dagger \dagger}$ The authors are with Academy of Systems Engineering, Academy of Military Sciences, Beijing 100141, China.

a) E-mail: paper_review@126.com

DOI: $10.1587 /$ transinf.2018EDL8016 behavior, in which the UASNs may be broken to be disconnected. Specially, the appearance and disappearance of dumb nodes are temporary and dynamic, as dumb node will become normal when environment changes to be favorable. The failure behavior about dumb node was not explicitly analyzed until to the Misra's study [1] in 2014. Although activating some redundant sleep nodes can effectively solve the topological holes, these redundant nodes will cause a waste of cost once dumb nodes are restored to normal. Therefore, it is very necessary to study how to detect dynamic dumb nodes, fix dumb nodes and guarantee the transmission of normal nodes without redundant nodes. The overall contributions of this work are as follows:

(a) The dual communication mode is designed. When the nodes become dumb, they can switch to lower frequency mode so as to transmit their stored data to farther distance with the same energy.

(b) The dumb-like nodes are defined and an adaptive connectivity re-establishment scheme is designed. When a parent node becomes dumb, the child nodes can quickly find a new parent node and transfer the entire child tree to achieve reliable data delivering without deploying redundant sleep nodes.

(c) Update the tree structure periodically to improve the network performance. When the dumb node returns to normal, UASNs can restore optimal structure quickly and achieve high efficiencies.

\section{Related Work}

The problem of dumb behavior of nodes were proposed originally in wireless sensor networks area. Researchers have proposed many techniques to address the connectivity reestablishment approach for dumb nodes. Misra [1] firstly defined dumb node and dumb behavior. It pointed out that dumb behavior is different from permanent failure. It is dynamic in nature. Roy [2] proposed a distributed approach for the detection of dumb nodes, named D3. It uses cumulative sum test to detect the dumb behavior. Kar [3] marks the neighbor nodes of the dumb node to isolate the dumb region. The dynamic avoidance routing protocol was designed to utilize the neighbor nodes for transmission. LECRAD scheme was proposed in [4]. The above scheme was mainly aimed at the WSN on land, and most of them considered the awakening of redundant sleep nodes to rebuild the relay, which was more expensive. At present, the healing studies in UASNs were mainly focused on the 
static holes. However, the dynamic holes, caused by dumb nodes or other factors, had attracted much attention in recent years. In this work, underwater dumb nodes are considered for the first time. A scheme named EECR is designed. It proposes dumb-node FM transmission and connectivity reestablishment method based on tree architecture.

\section{Problem Statement and Method Design}

As a classic topology control protocol in WSN, A1 protocol [5] can construct network connection topology easily and efficiently. Firstly, we use the A1 protocol to build a transport tree for UASNs. The surface sink is used as the root to initiate the tree construction. Taking the network shown in Fig. 1 (a) as an example, the dashed lines represent the adjacent relationship between the nodes. The root node $\mathrm{O}$ first marks itself as the 0th layer and broadcasts the Hello message, including its own layer. If node $\mathrm{O}$ receives the Hello responses before timeout then records these reply nodes as child nodes, otherwise mark itself as a leaf node. After receiving the Hello message from the root node, nodes A and I mark themselves as 1th layer and mark node $\mathrm{O}$ as their parent node. Then, they broadcast Hello messages to all the other neighbor nodes except the parent node O. Same as node $\mathrm{O}$, if nodes $\mathrm{A}$ and $\mathrm{I}$ receive the response before timeout then records their child nodes, otherwise mark themselves as leaf nodes. Likewise, nodes $\mathrm{B}$ and $\mathrm{C}$ will mark $\mathrm{A}$ as their own parent, and node $\mathrm{J}$ will marks I as its own parent. In particular, node D and node $\mathrm{E}$ will receive Hello messages from each other, and they are both at 3th level. So node E doesn't respond to node D and node D will mark itself as a leaf node. Repeating the above process will quickly get the tree topology shown in Fig. 1 (b) eventually.

\subsection{Problem Statement}

According to the actual characteristics of UASNs, nodes in the network can be divided into three types: normal node, dumb node and dumb-like node. Assuming $A_{i}$ as the neighbors set of node $i, S_{i}$ as the set of child nodes of $i, f_{i}$ as the parent node of $i$, and $r c_{i}(t)$ as the communication range at time $t$, each definition are given as follows:

Definition 1: Normal Node. Nodes which can sense and forward data normally. It can be expressed as:
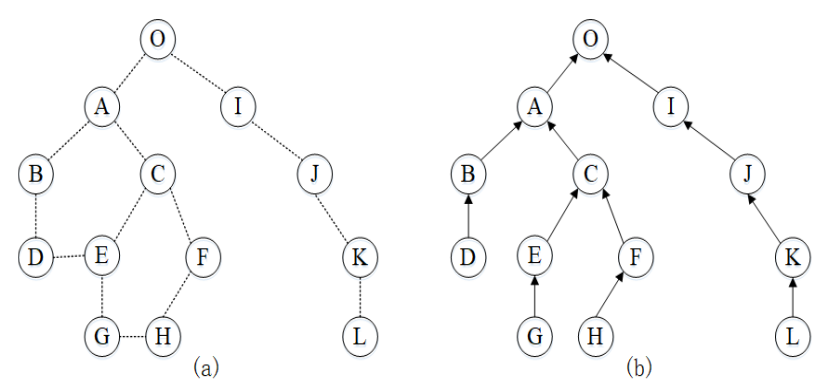

Fig. 1 Topology construction diagram with A1 protocol

$$
\Psi_{n}=\left\{\begin{array}{cc}
1, & \left(0<\min \left\{d_{i j} \mid j \in A_{i}\right\}<r c_{i}\left(t_{k}\right)\right) \\
0, & \text { otherwise }
\end{array}\right.
$$

Where $d_{i j}$ represents the distance between node $i$ and $j$. Node $i$ can behavior normally when $r c_{i}\left(t_{k}\right)$ is greater than the minimum distance between $i$ and its neighbors.

Definition 2: Dumb Node. Nodes which can perceive the physical surrounding, but can't forward data to any neighbor node. It can be expressed as:

$$
\Psi_{d}=\left\{\begin{array}{lc}
1, & \left(0<r c_{i}\left(t_{k}\right)<\min \left\{d_{i j} \mid j \in A_{i}\right\}\right) \\
0, & \text { otherwise }
\end{array}\right.
$$

That is, when $r c_{i}\left(t_{k}\right)$ is less than the minimum distance between $i$ and its neighbors, node $i$ becomes a dumb node.

Definition 3: Dumb-like Node. If parent node is a dumb node and the node has no neighbors except its child nodes, it becomes an isolated node called dumb-like node. It can be expressed as:

$$
\Psi_{d l}=\left\{\begin{array}{cc}
1, & 0<r c_{f_{i}}\left(t_{k}\right)<\min \left\{d_{f_{i}} \mid j \in A_{i}\right\} \& \& \\
& 0<r c_{i}\left(t_{k}\right)<\min \left\{d_{i j} \mid j \in\left(A_{i}-S_{i}-\left\{f_{i}\right\}\right)\right\} \\
0, & \text { otherwise }
\end{array}\right.
$$

Different from the failure of nodes, the dumb or dumblike node is only a kind of state, affected by a particular environment in particular time. When the environment restores favorable conditions, dumb nodes can be automatically converted to normal nodes. Therefore, to solve the connectivity problem caused by dumb or dumb-like nodes, we can't simply reconstruct the network in a fault-recovery manner, which may inevitably lead to unnecessary overhead.

\subsection{Method Design}

For the existence of dummy nodes topology, this paper presents EERC program, focusing on solving two problems. First, how does the current dumb node pass the information stored by itself? Second, how does the child node of the dumb node look for a new father and rebuild the reliable link? In the actual transfer process, the node will receive feedback from the parent node each time after the data sand.Therefore, in the premise of reliable communication, if a node $i$ sends data and receives no feedback from the parent, it will initiate a link-aware request to the other neighbor nodes. As a result, if node $i$ can communicate with other neighbor nodes except its child nodes, it will connect to the new parent node. If only the child nodes respond to the request, node $i$ will mark itself as a dumb-like node. If no response is received, node $i$ will mark itself as a dumb node. Using the change of node states as trigger conditions, the following mechanisms are designed to achieve rapid network recovery.

(a) dumb nodes FM transmission. Acoustic communication is the best choice for underwater wireless communication at present. However, the acoustic waves in different frequencies have different degrees of attenuation underwater. The path loss of the signal can be calculated as Eq. (4):

$$
A(d, f)=A_{0} d^{k} a(f)^{d}
$$


Where $A_{0}$ is normalized constant, $f$ is the signal frequency and $d$ is the transmission distance. The path-loss component, $k$, models the spreading loss and is usually between one and two. The absorption coefficient $a(f)$ can be obtained using Thorp empirical equation, as described in [6], and is expressed in Eq. (5).

$$
10 \log a(f)=\frac{0.11 f^{2}}{1+f^{2}}+\frac{44 f^{2}}{4100+f^{2}}+\frac{2.75 f^{2}}{10^{4}}+0.003
$$

It can be seen that the higher the frequency is, the faster the attenuation will be.Using attenuation $A(d, f)$ and noise power spectral density $N(f)$ calculated by $a(f)[6]$, the signal-to-noise ratio (SNR) at the distance $d$ can be calculated as follows:

$$
S N R(d, f)=\frac{S(f)}{A(d, f) N(f)}
$$

Where $S(f)$ is the power spectral density of the transmitted signal. It can be found that when the optimal signalto-noise ratio is reached, the more the acoustic communication frequency is, the further the designated transmission distance is. So, this paper defines different modes of communication for sensor nodes.

Model1: Normal communication. According to the distance between the nodes, we can select the optimal communication frequency for normal communication.

Model2: Dumb communication. When the node becomes dumb, lower communication frequency can extend communication distance. The currently perceived stored data can be sent to its parent node in the form of low frequency.

Although the bandwidth of dumb communication mode is low, its enough to support single node or few nodes to send the stored data out. By switching communication mode, dumb nodes can ensure the data perceived and stored successfully transmitted.

(b) Connectivity re-establishment for child nodes. Switching the communication modes can effectively ensure reliable transmission of their own data for the dumb and dumb-like nodes. However, the dumb-like node can not continue as a relay. It will broadcast a dumb-like message to its child nodes and switch its communication mode. The child nodes can quickly reconstruct communication links when they received the message. The algorithm for connectivity re-establishment is as follows:

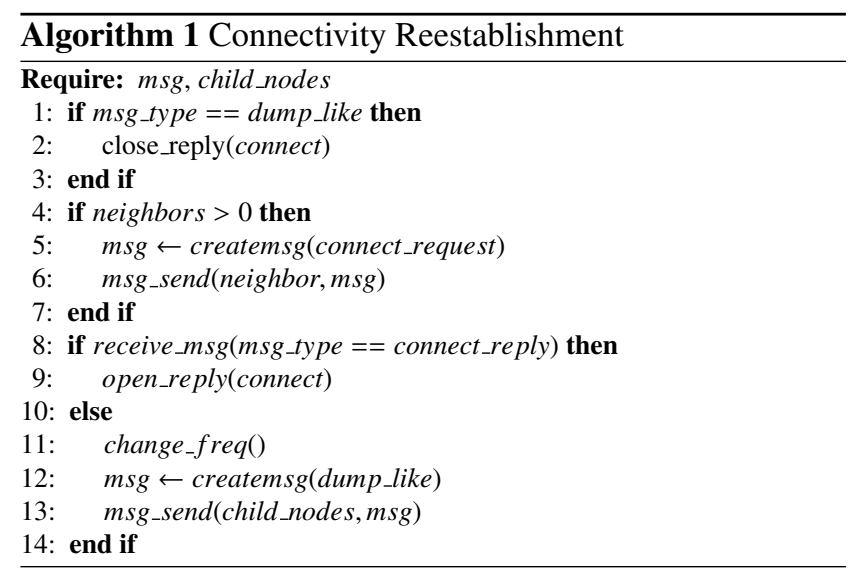

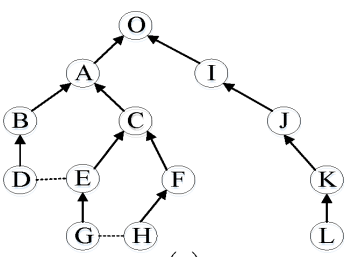

(a)

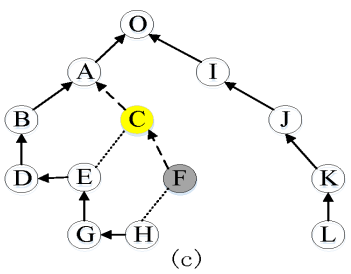

(c)

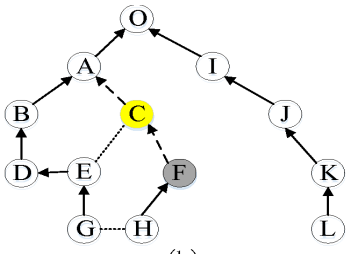

(b)

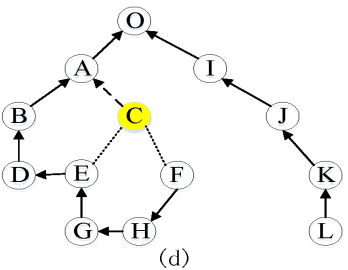

(d)
Fig. 2 The link of child nodes reconstruction process

Figure 2 (a) shows the best tree topology where solid arrows indicate normal communication connections and dashed lines indicate neighbors are reachable. When node $\mathrm{C}$ becomes a dumb node due to environmental impact, node $\mathrm{C}$ reduces the transmission frequency as described in Sect.3.2.1 to guarantee the delivery of its own data. Since node $\mathrm{C}$ becomes a dumb node, nodes $\mathrm{E}$ and $\mathrm{F}$ initiate link sensing because no feedback is received. Node E can sense the neighbor node $\mathrm{D}$ while node $\mathrm{F}$ cannot perceive other neighbor nodes except its child node. Therefore, node E initiates a connection request to node $\mathrm{D}$ and updates $\mathrm{D}$ as its new parent node after receiving the response. Node F marks itself as a dumb-like node, switches the communication mode and broadcasts a dumb-like message to its child nodes (line 9-13) (Fig. 2 (b)). After receiving the dumb-like message from the parent node $\mathrm{F}$, the child node $\mathrm{H}$ first disables its own connectable response (line 2) and broadcasts a connect request message (line3-5) to the neighbor nodes not including its parent and child nodes. Node $\mathrm{H}$ marks $\mathrm{G}$ as its new parent node after receiving the response from neighbor node G (line 6-7) (Fig. 2 (c)). After a certain period of time, node $\mathrm{F}$ recalculates the algorithm to request a neighbor connection, update $\mathrm{H}$ as its new parent node, and changes its state from dumb-like node to normal node. Finally the connectivity re-establishment process is finished (Fig. 2 (d)).

(c) Child tree updated. As the generation and restoration of dumb nodes according to the dynamic environment, when the dumb node senses the change of the external environment, it will try to communicate with its parent node using the normal frequency. If it has received the feedback from the parent node, the dumb node returns to normal. Since the tree structure constructed by the A1 protocol is the current optimal communication structure, when the dumb node is restored to normal, the tree structure needs to be restored. However, since the appearance of dumb nodes doesn't affect the entire topology (as shown in Fig. 2).The best tree structure shown in Fig. 2 (a) can be restored simply from the node $\mathrm{C}$. 


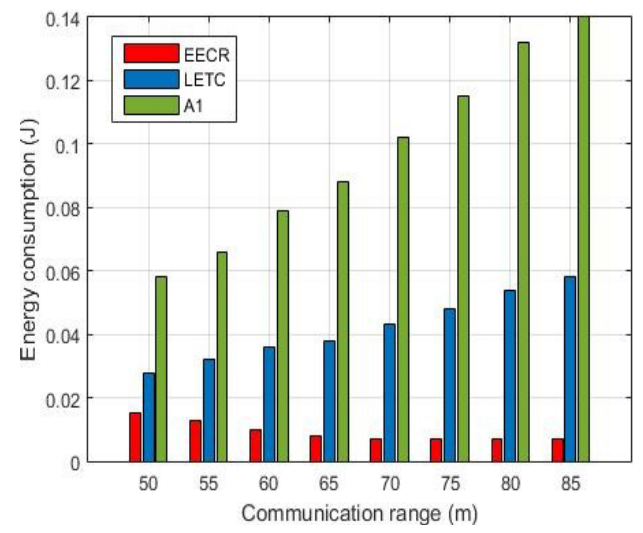

Fig. 3 Comparison of energy consumption

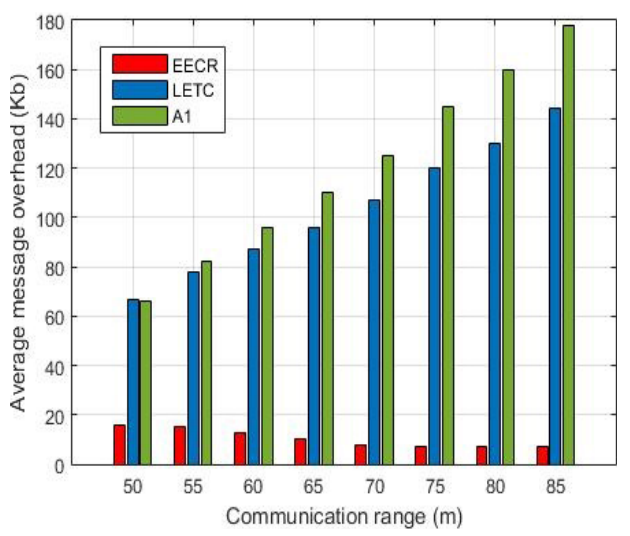

Fig. 4 Comparison of average message overhead

\subsection{Simulation Experiment}

In order to validate the functionality and accuracy of the EECR, an experimental platform for verification based on Omnet ++ has been set up. There are 200 nodes randomly deployed in the $500 * 500 \mathrm{~m}$ monitoring area. This paper compares EECR with LETC [7] and A1 [5] from three aspects: energy consumption, average message overhead and average throughput. Energy consumption refers to the cost during network connectivity re-establishment. The average message overhead represents the average cost of control messages. Average throughput refers to the amount of data received by the sink node in unit of time.

In Fig. 3 and Fig. 4, it can be found that with the increase of communication range, the energy consumption and average message overhead of EECR algorithm decrease. While the two indicators increase with the application of LETC and A1 algorithms. This is because both LETC and A1 algorithms take the network topology rebuilt from the beginning when the network topology is damaged. While, the EECT algorithm only rebuilds the subtree from the parent node of the dumb node, without the need to reconstruct the undamaged part.

Figure 5 shows that as the duration of the dumb behav-

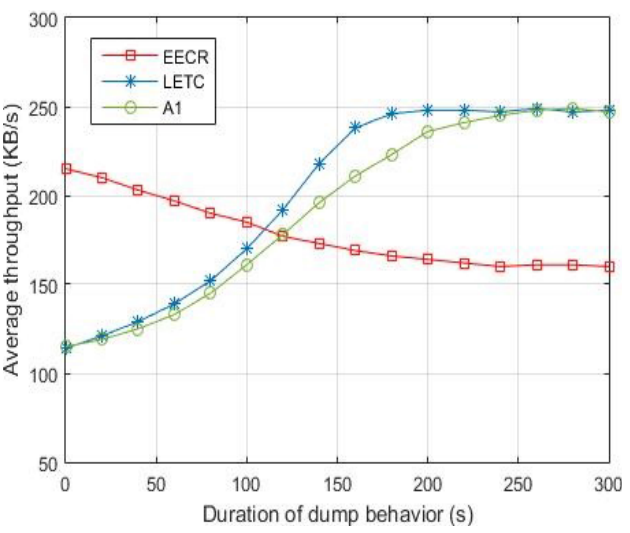

Fig. 5 Comparison of detection rate and false negative rate

ior increases, the average throughput under the EECR algorithm gradually decreases. The LETC and A1 algorithms have lower throughput rates with shorter dumb durations and higher throughput rates with longer dumb durations. The dynamic generation and disappearance of dumb nodes have the great influence on the EECR algorithm. Therefore, when the duration of the dumb behavior is short, the EECR keeps the communication maintained by the FM adjustment of the dumb node and the reconstruction of the subtree. Its cost is much less than that of all the topological reconstructions based on LETC and A1 algorithms. When the duration is longer, it is wise to rebuild the network topology for longterm transmission efficiency.

To improve the efficiency of EECR, a feasible solution is to add a global decision mechanism to it. That is, when the sink node discovers that the throughput rate has dropped within a certain limited time, the topology reconfiguration process should be started. This part of the content will be researched in the furture.

\section{Conclusion}

In this work, the special failure behavior of dumb node was considered, and the energy-efficient connectivity reestablishment (EECR) method was designed. This method deals with the data transmission of dumb nodes itself, the reconstruction of normal nodes connectivity, and the connection of isolated nodes caused by dumb nodes. Compared with the classical methods LETC and A1, the EECR has better performance in both message overhead and energy consumption, and has a better throughput under shorter duration dumb behavior. So in the future work, the problem of how to add a global decision mechanism based on EECR can be researched.

\section{Acknowledgments}

This work was supported by the Natural Science Foundation of Jiangsu Province under Grant No. BK20150721, BK20161469; China Postdoctoral Science Foundation under Grant No. 2015M582786, 2016T91017; En- 
gineering Research Center of Jiangsu Province under Grant No. BM2014391; Primary Research \& Development Plan of Jiangsu Province under Grant No. BE2015728, BE2016904, BE2017616; National Key Research and Development Program No.2016YFC0800606, 2016YFC0800310; Key Consulting Project of Chinese Academy of Engineering under Grant No.2017-XZ-05.

\section{References}

[1] S. Misra, P. Kar, A. Roy, and M.S. Obaidat, "Existence of dumb nodes in stationary wireless sensor networks," J. Systems and Software, vol.91, no.1, pp.135-146, May 2014.

[2] A. Roy, P. Kar, S. Misra, and M.S. Obaidat, "D3: Distributed approach for the detection of dumb nodes in wireless sensor networks," Int. J. Communication Systems, vol.30, no.1, pp.799-813, Jan. 2017.
[3] P. Kar and S. Misra, "Detouring dynamic routing holes in stationary wireless sensor networks in the presence of temporarily misbehaving nodes," Int. J. Communication Systems, vol.30, no.4, pp.1-17, March 2017.

[4] P. Kar, A. Roy, S. Misra, and M.S. Obaidat, "Energy-efficient connectivity re-establishment in WSN in the presence of dumb nodes," IEEE International Conference on Communication Workshop, pp.14851490, IEEE, 2015.

[5] S. Rizvi, H.K. Qureshi, S.A. Khayam, V. Rakocevic, and M. Rajarajan, "A1: An energy efficient topology control algorithm for connected area coverage in wireless sensor networks" J. Network and Computer Applications, vol.35, no.2, pp.597-605, March 2012.

[6] S. Climent, A. Sanchez, J.V. Capella, N. Meratnia, and J.J. Serrano, "Underwater acoustic wireless sensor networks: Advances and future trends in physical, MAC and routing layers," Sensors, vol.14, no.1, pp.795-833, 2014.

[7] J.A. Torkestani, "An energy-efficient topology construction algorithm for wireless sensor networks," Computer Networks, vol.57, no.7, pp.1714-1725, May 2013. 